\title{
Entire Functions with Separated Zeros and 1-Points
}

\author{
Walter Bergweiler ${ }^{1} \cdot$ Alexandre Eremenko $^{2}$
}

Received: 2 February 2020 / Accepted: 20 August 2020 / Published online: 14 September 2020

(c) The Author(s) 2020

\section{Abstract}

We consider transcendental entire functions of finite order for which the zeros and 1-points are in disjoint sectors. Under suitable hypotheses on the sizes of these sectors we show that such functions must have a specific form, or that such functions do not exist at all.

Keywords Entire function - Value distribution - Sector - Radially distributed value · Subharmonic function

Mathematics Subject Classification 30D20 · 30D35

\section{Introduction and Results}

Our starting point is the following result of Biernacki [3, p. 533].

Theorem A There is no transcendental entire function of finite order for which the zeros accumulate in one direction and the 1-points accumulate in a different direction. Here we say that a set $\left\{a_{n}\right\}$ of complex numbers accumulates in one direction if there exists a ray such that for every open sector bisected by this ray all but finitely many $a_{n}$ lie in this sector.

\section{Dedicated to the memory of Stephan Ruscheweyh}

Communicated by Filippo Bracci.

Supported by NSF Grant DMS-1665115.

$凶$ Walter Bergweiler

bergweiler@math.uni-kiel.de

Alexandre Eremenko

eremenko@math.purdue.edu

1 Mathematisches Seminar, Christian-Albrechts-Universität zu Kiel, Ludewig-Meyn-Str. 4, 24098

Kiel, Germany

2 Department of Mathematics, Purdue University, West Lafayette, IN 47907, USA 
We will prove the following generalizations of Theorem A.

Theorem 1.1 Let $S_{0}$ and $S_{1}$ be closed sectors in $\mathbb{C}$ satisfying $S_{0} \cap S_{1}=\{0\}$. Let $\theta_{j}$ denote the opening angle of $S_{j}$ and suppose that

$$
\min \left\{\theta_{0}, \theta_{1}\right\}<\frac{\pi}{2} \text { and } \max \left\{\theta_{0}, \theta_{1}\right\}<\pi
$$

Then there is no transcendental entire function of finite order for which all but finitely many zeros are in $S_{0}$ while all but finitely many 1-points are in $S_{1}$.

Theorem 1.2 Let $S$ be a closed sector in $\mathbb{C}$ of opening angle less than $\pi / 3$ and let $H$ be a closed half-plane intersecting $S$ only in 0 . Let $f$ be a transcendental entire function of finite order. Suppose that all but finitely many zeros of $f$ are in $S$ while all but finitely many 1-points are in $H$. Then $f$ has the form $f(z)=P(z) e^{a z}$ where $P$ is a polynomial and $a \in \mathbb{C}$.

The following examples show that the hypotheses of Theorems 1.1 and 1.2 are sharp. We will verify in Sect. 2 that these examples have the stated properties.

Our first example shows that the condition $\min \left\{\theta_{0}, \theta_{1}\right\}<\pi / 2$ in Theorem 1.1 cannot be relaxed to $\min \left\{\theta_{0}, \theta_{1}\right\} \leq \pi / 2$.

Example 1.1 Let

$$
f(z):=\frac{2}{\sqrt{\pi}} \int_{0}^{z} t^{2} \exp \left(-t^{2}\right) d t+\frac{1}{2}
$$

Then all but finitely many zeros of $f$ are in $\{z:|\arg z| \leq \pi / 4\}$ while all but finitely many 1-points of $f$ are in $\{z:|\arg z-\pi| \leq \pi / 4\}$.

The exponential function shows that the condition $\max \left\{\theta_{0}, \theta_{1}\right\}<\pi$ in Theorem 1.1 cannot be relaxed to $\max \left\{\theta_{0}, \theta_{1}\right\} \leq \pi$. This is also shown by the following example, which has infinitely many zeros and 1-points. This example also shows that the conclusion of Theorem 1.2 does not hold if $S$ has opening angle equal to $\pi / 3$.

Example 1.2 Let $a$ and $b$ be defined by

$$
a \int_{0}^{\infty} t^{3} \exp \left(-t^{3}\right) d t=\frac{1}{3} \text { and } b \int_{0}^{\infty} t \exp \left(-t^{3}\right) d t=\frac{1}{3}
$$

Let

$$
f(z):=\int_{0}^{z}\left(a t^{3}+b t\right) \exp \left(-t^{3}\right) d t+\frac{1}{3}
$$

Then all but finitely many zeros of $f$ are in $\{z:|\arg z| \leq \pi / 6\}$ while all but finitely many 1-points of $f$ are in $\{z: \operatorname{Re} z \leq 0\}$.

Theorems 1.1 and 1.2 will be derived from the following result. 
Theorem 1.3 Let $S_{0}$ and $S_{1}$ be closed sectors in $\mathbb{C}$ of opening angles at most $\pi$. Suppose that $S_{0} \cap S_{1}=\{0\}$ and let $f$ be a transcendental entire function of finite order for which all but finitely many zeros are in $S_{0}$ while all but finitely many 1-points are in $S_{1}$. Then $f$ has the form

$$
f(z)=\int_{0}^{z} p(\zeta) e^{q(\zeta)} d \zeta+c
$$

where $p$ and $q$ are polynomials and $c \in \mathbb{C}$.

The conclusion of Theorem 1.3 does not hold if one of the sectors $S_{0}$ and $S_{1}$ has opening angle greater than $\pi$. Similarly, the half-plane in Theorem 1.2 cannot be replaced by a sector of opening angle greater than $\pi$.

For example, if $0<\rho<1$ and $\left(a_{n}\right)$ is a sequence of positive real numbers satisfying $a_{n} \sim n^{1 / \rho}$ as $n \rightarrow \infty$, then

$$
f(z):=\prod_{n=1}^{\infty}\left(1-\frac{z}{a_{n}}\right)
$$

is an entire function with positive zeros for which the 1-points accumulate at the rays $\arg z= \pm \pi(1-1 / 2 \rho)$; see, e.g., [8, Sect. 2.5] for the asymptotics of canonical products with positive zeros.

The restriction on the order is essential in all stated theorems. In fact, for any two distinct directions there exists an entire function $f$ of infinite order such that the zeros of $f$ accumulate in one of these directions while the 1-points accumulate in the other direction; see, e.g., [2, Thm. 5].

On the other hand, a classical result of Edrei [5] says that if the zeros and 1-points of an entire function $f$ lie on finitely many rays, then $f$ has finite order.

\section{Examples}

We consider functions $f$ of the form (1.1). Let $d:=\operatorname{deg}(q)$ and let $A$ be the leading coefficient of $q$ so that $q(z) \sim A z^{d}$ as $z \rightarrow \infty$. For $k \in\{1,2, \ldots, d\}$ we put

$$
\phi_{k}:=\frac{(2 k-1) \pi-\arg A}{d} .
$$

It is easy to see that the limits

$$
a_{k}:=\lim _{r \rightarrow \infty} f\left(r e^{i \phi_{k}}\right)
$$

exist. For $\varepsilon>0$ we then have, as $|z| \rightarrow \infty$,

$$
f(z) \rightarrow a_{k} \text { for } \phi_{k}-\frac{\pi}{2 d}+\varepsilon \leq \arg z \leq \phi_{k}+\frac{\pi}{2 d}-\varepsilon
$$


while

$$
|f(z)| \rightarrow \infty \text { for } \phi_{k}+\frac{\pi}{2 d}+\varepsilon \leq \arg z \leq \phi_{k+1}-\frac{\pi}{2 d}-\varepsilon
$$

Here we have put $\phi_{d+1}=\phi_{1}+2 \pi$.

The following quantitative form of (2.3) and (2.4) can be proved using integration by parts; see [10, Lem. 4.1].

\section{Lemma 2.1 Let}

$$
\phi_{k}-\frac{\pi}{d} \leq \arg z \leq \phi_{k}+\frac{\pi}{d}
$$

Then

$$
f(z)=a_{k}+\frac{p(z)}{q^{\prime}(z)} e^{q(z)}\left(1+\mathcal{O}\left(\frac{1}{|z|}\right)\right)
$$

as $|z| \rightarrow \infty$.

It is well-known - and follows easily from Lemma 2.1 - that for any $a \in \mathbb{C} \backslash\left\{a_{k}\right\}$ and $\varepsilon \in(0, \pi / d)$ each of the sectors $\left\{z:\left|\arg z-\phi_{k} \pm \pi /(2 d)\right|<\varepsilon\right\}$ contains infinitely many $a$-points, but only finitely many $a_{k}$-points.

Thus for any $a \in \mathbb{C}$ the $a$-points of $f$ can accumulate only at the rays given by $\arg z=\phi_{k} \pm \pi /(2 d)$, and they do accumulate at the rays $\arg z=\phi_{k} \pm \pi /(2 d)$ for $a \in \mathbb{C} \backslash\left\{a_{k}\right\}$, but not for $a=a_{k}$.

\section{Verification of Example 1.1 Since}

$$
\int_{0}^{\infty} t^{2} \exp \left(-t^{2}\right) d t=\frac{1}{4} \sqrt{\pi}
$$

we have (2.2) with $\phi_{1}=0, \phi_{2}=\pi, a_{1}=1$ and $a_{2}=0$. Thus the zeros are asymptotic to the rays $\arg z= \pm \pi / 4$ while the 1-points are asymptotic to the rays $\arg z= \pm 3 \pi / 4$. Lemma 2.1 yields that $|f(z)| \rightarrow \infty$ as $|z| \rightarrow \infty$ if $\pi / 4 \leq \arg z \leq$ $3 \pi / 4$ or $-3 \pi / 4 \leq \arg z \leq-\pi / 4$. This implies that all but finitely many zeros are contained in $\{z:|\arg z|<\pi / 4\}$ and all but finitely many 1-points are contained in $\{z:|\arg z-\pi|<\pi / 4\}$.

Verification of Example 1.2 Here we have $d=3$ and (2.2) holds with $\phi_{1}=0, \phi_{2}=$ $2 \pi / 3, \phi_{3}=4 \pi / 3, a_{1}=1$ and $a_{2}=a_{3}=0$. Thus the zeros are asymptotic to the rays $\arg z= \pm \pi / 6$ while the 1 -points are asymptotic to the rays $\arg z= \pm \pi / 2$ and $\arg z= \pm 5 \pi / 6$. The rest of the argument follows as in Example 1.1 from Lemma 2.1.

\section{Preliminary Results}

We shall need some results about subharmonic functions; see [9], [11, Ch. III], [12, Ch. III], [14] for basic results used in the following. 
The following result is implicit in [2]. For completeness, we include a proof.

Lemma 3.1 Let $u$ be subharmonic function in a neighborhood of 0 and $u(0)=0$. Let $\alpha \in(0, \pi]$ and suppose that $u(z)<0$ for $|\arg z|<\alpha$. Then $\alpha \leq \pi / 2$, and there exists $c>0$ and $r_{0}>0$ such that

$$
\int_{-\alpha}^{\alpha} u\left(r e^{i t}\right) d t \leq-c r^{\pi /(2 \alpha)} \text { for } r \in\left(0, r_{0}\right)
$$

Proof Let $r_{1}>0$ be such that the closed disk of radius $r_{1}$ around 0 is in the domain of $u$. Then there exists a harmonic majorant $h$ of $u$ in the (truncated) sector $S_{\alpha}:=$ $\left\{z:|\arg z|<\alpha,|z|<r_{1}\right\}$ such that $h\left(r e^{ \pm i \alpha}\right)=0$ for $r \in\left(0, r_{1}\right)$. Let $r_{2}:=r_{1}^{\pi /(2 \alpha)}$ and $S_{\pi / 2}:=\left\{z: \operatorname{Re} z>0,|z|<r_{2}\right\}$. Then the function $v$ defined by $v(z)=h\left(z^{2 \alpha / \pi}\right)$, where the principal branch of the root is used, is negative and harmonic in $S_{\pi / 2}$, and zero on the vertical part of the boundary. By the reflection principle $v$ extends to a harmonic function in the disc of radius $r_{2}$ around 0 , and $\nabla v(0) \neq 0$. Therefore $v(z)=-c_{0} \operatorname{Re} z+\mathcal{O}\left(z^{2}\right)$ near zero for some $c_{0}>0$. Thus

$$
\int_{-\pi / 2}^{\pi / 2} v\left(r e^{i t}\right) d t \leq-\frac{1}{2} c_{0} r
$$

if $r$ is small enough. This implies that (3.1) is satisfied with $h$ instead of $u$. Since $u<h$ in $S_{\alpha}$, we deduce that $u$ also satisfies (3.1).

To show that $\alpha \leq \pi / 2$, let $S_{\alpha}^{\prime}=\left\{z: \alpha<\arg z<2 \pi-\alpha,|z|<r_{1}\right\}$ be the complement of $\overline{S_{\alpha}}$ in the disk of radius $r_{1}$ and let $h_{1}$ be a harmonic majorant of $u$ in $S_{\alpha}^{\prime}$ which satisfies $h_{1}\left(r e^{ \pm i \alpha}\right)=0$ for $r \in\left(0, r_{1}\right)$. The same argument as before shows that $h_{1}(z)=\mathcal{O}\left(z^{\pi /(2 \beta)}\right)$ as $z \rightarrow 0$, where $\beta:=\pi-\alpha$. Thus there exists a constant $c_{1}>0$ such that

$$
\int_{\alpha}^{2 \pi-\alpha} u\left(r e^{i t}\right) d t \leq c_{1} r^{\pi /(2 \beta)} \text { for } r \in\left(0, r_{0}\right)
$$

Together with (3.1) this yields that

$$
0=u(0) \leq \int_{\alpha}^{2 \pi+\alpha} u\left(r e^{i t}\right) d t \leq c_{1} r^{\pi /(2 \beta)}-c r^{\pi /(2 \alpha)} \text { for } r \in\left(0, r_{0}\right)
$$

We conclude that $\beta \geq \alpha$ and thus $\alpha \leq \pi / 2$.

In the case that $\alpha=\pi / 2$ we will use the following result.

Lemma 3.2 Let $u$ be subharmonic in $\mathbb{C}$. Suppose that $u(0)=0$ and that there exist $\rho>0$ and $K>0$ such that $u(z) \leq K|z|^{\rho}$ for all $z \in \mathbb{C}$. If $u$ is negative in some half-plane, then $\rho=1$.

In the proof of Lemma 3.2 we will use the following version of the Phragmén-Lindelöf theorem [14, Cor. 2.3.8]. 
Lemma 3.3 Let $u$ be subharmonic in the right half-plane $H:=\{z: \operatorname{Re} z>0\}$. Suppose that there exist constants $A, B \in \mathbb{R}$ such that

$$
u(z) \leq A+B|z| \text { for } z \in H
$$

and

$$
\limsup _{\zeta \rightarrow z} u(\zeta) \leq 0 \text { for } z \in \partial H
$$

Put

$$
L:=\limsup _{x \rightarrow \infty} \frac{u(x)}{x}
$$

Then

$$
u(z) \leq L \operatorname{Re} z \text { for } z \in H
$$

Proof of Lemma 3.2 Without loss of generality we may assume that $u(z)<0$ for $\operatorname{Re} z<0$. Lemma 3.1 and the hypotheses that $u(0)=0$ and $u(z) \leq K|z|^{\rho}$ yield that if $r$ is sufficiently small, then

$0=2 \pi u(0) \leq \int_{-\pi / 2}^{3 \pi / 2} u\left(r e^{i t}\right) d t=\int_{-\pi / 2}^{\pi / 2} u\left(r e^{i t}\right) d t+\int_{\pi / 2}^{3 \pi / 2} u\left(r e^{i t}\right) d t \leq K \pi r^{\rho}-c r$.

This yields that $\rho \leq 1$. Hence (3.2) holds with $A=B=K$.

Lemma 3.3 yields that (3.4) holds with $L$ given by (3.3). Since $u$ is non-constant and hence unbounded we have $L>0$. Thus $\rho=1$.

Let $u$ be subharmonic in $\mathbb{C}$ and let $\mu$ be the Riesz measure of $u$. For $r>0$ we put

$$
B(r, u):=\max _{|z|=r} u(z) \text { and } n(r):=\mu(\{z:|z| \leq r\}) .
$$

Jensen's formula (see [13, Sect. 7.2] or [9, Sect. 3.9]) yields that

$$
N(r):=\int_{1}^{r} \frac{n(t)}{t} d t \leq B(r, u)+\mathcal{O}(1)
$$

The order $\rho$ of $u$ is defined by

$$
\rho:=\limsup _{r \rightarrow \infty} \frac{\log B(r, u)}{\log r}
$$

Note that if $u=\log |f|$ for some entire function $f$, and if $M(r)$ denotes the maximum modulus of $f$, then we have $B(r, u)=\log M(r)$. Thus the order of the subharmonic function $u$ coincides with that of the entire function $f$. 
If $u$ has finite order, then there exists a non-negative integer $q$ satisfying $q \leq \rho \leq$ $q+1$ such that

$$
\int_{1}^{\infty} \frac{B(r, u)}{r^{q+2}} d r<\infty .
$$

Using (3.5) we also see that

$$
\int_{1}^{\infty} \frac{N(r)}{r^{q+2}} d r<\infty
$$

Moreover, integration by parts shows that the latter condition is equivalent to

$$
\int_{1}^{\infty} \frac{n(r)}{r^{q+2}} d r<\infty .
$$

The following result [9, Thm. 4.2] is the subharmonic version of the Hadamard factorization theorem.

Lemma 3.4 Let $u$ be subharmonic of finite order $\rho$ with Riesz measure $\mu$. Let $q$ be the minimal integer such that (3.6) holds and let $R>0$. Then $u$ can be written in the form

$$
u(z)=v(z)+w(z)+h(z)
$$

where

$$
\begin{aligned}
& v(z)=\int_{|\zeta|<R} \log |z-\zeta| d \mu(\zeta) \\
& w(z)=\int_{|\zeta| \geq R}\left(\log \left|1-\frac{z}{\zeta}\right|+\sum_{j=1}^{q} \frac{1}{j} \operatorname{Re}\left(\frac{z}{\zeta}\right)\right) d \mu(\zeta)
\end{aligned}
$$

and $h$ is a harmonic polynomial of degree at most $\rho$.

We also note that the function $w$ defined by (3.8) satisfies

$$
B(r, w)=o\left(r^{q+1}\right)
$$

as $r \rightarrow \infty$. This follows easily from [9, Lem. 4.4]; see also [8, p. 57, Rem. 2].

Lemma 3.5 Let $u$ be a subharmonic function of order at most 1 . Suppose that there exists $R>0$ and $\varepsilon>0$ such that $u$ is harmonic in each of the two domains $T_{ \pm}:=$ $\{z:|z|>R,|\arg z \pm \pi / 2|<\varepsilon\}$. Suppose also that $u$ is bounded on the imaginary axis. Then $u$ is harmonic and of the form $u(z)=a \operatorname{Re} z+b$ with $a, b \in \mathbb{R}$. 
Proof We apply Lemma 3.4. Since $u$ has order 1 , we have $q \in\{0,1\}$ in (3.8) and $h$ has the form

$$
h(z)=\operatorname{Re}(A z+B)
$$

for some $A, B \in \mathbb{C}$. For $y \in \mathbb{R}$ we then have

$$
h(i y)+h(-i y)=\operatorname{Re}(A i y+B)+\operatorname{Re}(-A i y+B)=2 \operatorname{Re} B
$$

Assuming that $u$ is not harmonic, we may choose $R$ such that $\mu(\{z:|z|<R\})$ is positive and we find that $v(z) \rightarrow \infty$ as $|z| \rightarrow \infty$. In particular,

$$
v(i y)+v(-i y) \rightarrow \infty
$$

as $y \rightarrow \infty$. Since $u$ is bounded on the imaginary axis, (3.7), (3.10) and (3.11) imply that

$$
Q(y):=w(i y)+w(-i y) \rightarrow-\infty
$$

First we consider the case $q=0$. Define $w^{*}$ by $w^{*}(z)=w(z)+w(\bar{z})$. Then $B\left(r, w^{*}\right)=o(r)$ as $r \rightarrow \infty$ by (3.9). Moreover, $w^{*}(i y)=Q(y)$ so that $w^{*}$ is bounded on the imaginary axis by (3.12). The Phragmén-Lindelöf theorem (Lemma 3.3) now implies that $w^{*}$ is constant, contradicting (3.12).

Now we consider the case $q=1$. In order to estimate $Q(y)$ in this case we note that

$$
\begin{aligned}
& \log \left|1-\frac{i y}{\zeta}\right|+\operatorname{Re}\left(\frac{i y}{\zeta}\right)+\log \left|1-\frac{-i y}{\zeta}\right|+\operatorname{Re}\left(\frac{-i y}{\zeta}\right) \\
& =\log \left|1-\frac{i y}{\zeta}\right|+\log \left|1-\frac{i y}{\bar{\zeta}}\right| \\
& =\log \left|1-i \frac{2 y \operatorname{Re} \zeta}{|\zeta|^{2}}-\frac{y^{2}}{|\zeta|^{2}}\right| \\
& =\frac{1}{2} \log \left(\left(1-\frac{y^{2}}{|\zeta|^{2}}\right)^{2}+4 \frac{y^{2}(\operatorname{Re} \zeta)^{2}}{|\zeta|^{4}}\right)
\end{aligned}
$$

so that

$$
Q(y)=\frac{1}{2} \int_{|\zeta| \geq R} \log \left(\left(1-\frac{y^{2}}{|\zeta|^{2}}\right)^{2}+4 \frac{y^{2}(\operatorname{Re} \zeta)^{2}}{|\zeta|^{4}}\right) d \mu(\zeta)
$$


For $|\arg \zeta \pm \pi / 2| \geq \varepsilon$ we have $|\operatorname{Re} \zeta| \geq \alpha|\zeta|$ with $\alpha:=\cos (\pi / 2-\varepsilon)>0$ and thus

$$
\begin{aligned}
\log \left(\left(1-\frac{y^{2}}{|\zeta|^{2}}\right)^{2}+4 \frac{y^{2}(\operatorname{Re} \zeta)^{2}}{|\zeta|^{4}}\right) & \geq \log \left(\left(1-\frac{y^{2}}{|\zeta|^{2}}\right)^{2}+4 \alpha^{2} \frac{y^{2}}{|\zeta|^{2}}\right) \\
& =\log \left(1+\left(4 \alpha^{2}-2\right) \frac{y^{2}}{|\zeta|^{2}}+\frac{y^{4}}{|\zeta|^{4}}\right)
\end{aligned}
$$

As $u$ is harmonic in $T_{ \pm}$we find that (3.14) holds for every $\zeta$ in the support of $\mu$ which satisfies $|\zeta| \geq R$. We put $n_{R}(r)=\mu\{z: R \leq|z| \leq r\}$ and note that $n_{R}(r)=$ $n(r)-\mu(\{z:|z|<R\})$; that is, $n_{R}(r)$ and $n(r)$ differ only by a constant. We deduce from (3.13) and (3.14), using also integration by parts, that

$$
\begin{aligned}
Q(y) & \geq \frac{1}{2} \int_{R}^{\infty} \log \left(1+\left(4 \alpha^{2}-2\right) \frac{t^{2}}{|\zeta|^{2}}+\frac{t^{4}}{|\zeta|^{4}}\right) d n(t) \\
& =2 \int_{0}^{\infty} n_{R}(t) K\left(\frac{y}{t}\right) \frac{d t}{t},
\end{aligned}
$$

where

$$
K(x):=\frac{x^{2}\left(2 \alpha^{2}-1+x^{2}\right)}{1+\left(4 \alpha^{2}-2\right) x^{2}+x^{4}}=\frac{x^{2}\left(\beta+x^{2}\right)}{1+2 \beta x^{2}+x^{4}}
$$

with $\beta:=2 \alpha^{2}-1=2 \cos ^{2}(\pi / 2-\varepsilon)-1=\cos (\pi-2 \varepsilon)$. It is easy to see that there exists a constant $c_{1}$ depending only on $\alpha$ such that

$$
|K(x)| \leq c_{1} x^{2} \text { for } x \geq 0
$$

In fact, this holds with $c_{1}:=1 /\left(4 \alpha \sqrt{1-\alpha^{2}}\right)$. Moreover, we have

$$
|K(x)| \leq \frac{4 x^{2}}{2+x^{4}} \text { for } x \geq 2 .
$$

By (3.12) there exists $y_{0}>0$ such that $Q(y) \leq 0$ for $y \geq y_{0}$. For $0<\delta<1$ we thus have

$$
\begin{aligned}
0 & \geq \int_{y_{0}}^{\infty} \frac{1}{y^{2+\delta}} Q(y) d y \\
& =\int_{y_{0}}^{\infty} \frac{1}{y^{2+\delta}} \int_{0}^{\infty} n_{R}(t) K\left(\frac{y}{t}\right) \frac{d t}{t} d y \\
& =\int_{0}^{\infty} n_{R}(t) \int_{y_{0}}^{\infty} \frac{1}{y^{2+\delta}} K\left(\frac{y}{t}\right) \frac{1}{t} d y d t \\
& =\int_{0}^{\infty} \frac{n_{R}(t)}{t^{2+\delta}} \int_{y_{0} / t}^{\infty} \frac{K(s)}{s^{2+\delta}} d s d t .
\end{aligned}
$$


Here we have changed the order of integration. This is justified by the Fubini-Tonelli theorem, since by $(3.15)$ and (3.16) the above integrals are finite if $K(\cdot)$ is replaced by $|K(\cdot)|$.

It follows from (3.15) that

$$
\int_{0}^{y_{0} / t} \frac{K(s)}{s^{2+\delta}} d s \leq c_{1} \int_{0}^{y_{0} / t} \frac{d s}{s^{\delta}}=\frac{c_{1}}{1-\delta}\left(\frac{y_{0}}{t}\right)^{1-\delta}
$$

and hence

$$
\int_{0}^{\infty} \frac{n_{R}(t)}{t^{2+\delta}} \int_{0}^{y_{0} / t} \frac{K(s)}{s^{2+\delta}} d s d t \leq c_{2}:=\frac{c_{1} y_{0}^{1-\delta}}{1-\delta} \int_{0}^{\infty} \frac{n_{R}(t)}{t^{3}} d t<\infty
$$

by (3.6). Combining this with (3.17) we deduce that

$$
\int_{0}^{\infty} \frac{n_{R}(t)}{t^{2+\delta}} d t \cdot \int_{0}^{\infty} \frac{K(s)}{s^{2+\delta}} d s \leq c_{2}
$$

for some constant $c_{2}$. Note that $c_{2}$ remains bounded as $\delta \rightarrow 0$.

We have

$$
\int_{0}^{\infty} \frac{K(s)}{s^{2+\delta}} d s=\frac{1}{2} \int_{0}^{\infty} x^{-\gamma} \frac{x+\beta}{1+2 \beta x+x^{2}} d x
$$

with $\gamma:=(1+\delta) / 2$. The computation of the integral on the right hand side is a standard application of the residue theorem which yields that

$\int_{0}^{\infty} \frac{K(s)}{s^{2+\delta}} d s=\frac{1}{2} \frac{\pi}{\sin (\pi \gamma)} \cos (\gamma(\pi-\arccos (-\beta)))=\frac{1}{2} \frac{\pi}{\sin (\pi \gamma)} \cos (\gamma(\pi-2 \varepsilon))$.

Since $\gamma \rightarrow 1 / 2$ as $\delta \rightarrow 0$ we thus have

$$
\lim _{\delta \rightarrow 0} \int_{0}^{\infty} \frac{K(s)}{s^{2+\delta}} d s=\frac{1}{2} \frac{\pi}{\sin (\pi / 2)} \cos \left(\frac{1}{2} \pi-\varepsilon\right)=\frac{1}{2} \pi \alpha>0 .
$$

Moreover,

$$
\int_{0}^{\infty} \frac{n_{R}(t)}{t^{2}} d t=\infty
$$

since $q=1$, and $q$ is chosen as the smallest integer such that (3.6) holds. Thus

$$
\lim _{\delta \rightarrow 0} \int_{0}^{\infty} \frac{n_{R}(t)}{t^{2+\delta}} d t=\infty
$$

Taking the limit as $\delta \rightarrow 0$ in (3.18) yields a contradiction to (3.19) and (3.20). 


\section{Proofs of the Theorems}

Proof of Theorem 1.3 Let $\theta_{j}$ be the opening angle of $S_{j}$. Since only one of the two sectors can have opening angle equal to $\pi$, we may assume without loss of generality that $\theta_{0}<\pi$. We may also assume that $f(0) \notin\{0,1\}$.

First we show that the genus of $f$ is at least 1 . Suppose that this is not the case so that $f$ is of genus 0 . Then $f$ has the form

$$
f(z)=f(0) \lim _{n \rightarrow \infty} \prod_{k=1}^{n}\left(1-\frac{z}{a_{k}}\right)=1+(f(0)-1) \lim _{n \rightarrow \infty} \prod_{k=1}^{n}\left(1-\frac{z}{b_{k}}\right),
$$

with all but finitely many $a_{k}$ contained in $S_{0}$ and all but finitely many $b_{k}$ contained in $S_{1}$. The Gauss-Lucas theorem and Hurwitz's theorem imply that all zeros of $f^{\prime}$ are contained in the convex hull of the $a_{k}$ as well as in the convex hull of the $b_{k}$. Thus $f^{\prime}$ has only finitely many zeros. Since we assumed that $f$ has genus 0 , this contradicts our hypothesis that $f$ is transcendental. Hence $f$ has genus at least 1 .

Let $\rho$ be the order of $f$. Since $f$ has genus at least 1 we have $\rho \geq 1$. Let $M(r)$ denote the maximum modulus of $f$. We proceed as in [2] and note that since $f$ is of finite order, there exists a sequence $\left(r_{k}\right)$ tending to $\infty$ such that

$$
\log M\left(t r_{k}\right)=\mathcal{O}\left(\log M\left(r_{k}\right)\right) \quad \text { as } k \rightarrow \infty
$$

for every $t>1$. The existence of such a sequence $\left(r_{k}\right)$ for a function $f$ of finite order is well-known and easy to prove, but it is also an immediate consequence of a result of Drasin and Shea [4] on the existence of Pólya peaks. Put

$$
\rho^{*}:=\sup \left\{p \in \mathbb{R}: \limsup _{r, t \rightarrow \infty} \frac{\log M(t r)}{t^{p} \log M(r)}=\infty\right\}
$$

and

$$
\rho_{*}:=\inf \left\{p \in \mathbb{R}: \liminf _{r, t \rightarrow \infty} \frac{\log M(t r)}{t^{p} \log M(r)}=0\right\} .
$$

The result of Drasin and Shea [4] says that

$$
0 \leq \rho_{*} \leq \rho \leq \rho^{*} \leq \infty
$$

and if $\lambda \in \mathbb{R}$ satisfies $\rho_{*} \leq \lambda \leq \rho^{*}$, then $\log M(r)$ has a sequence of Pólya peaks of order $\lambda$; that is, there exists a sequence $\left(r_{k}\right)$ tending to $\infty$ such that if $\varepsilon>0$, then

$$
\log M\left(t r_{k}\right) \leq(1+\varepsilon) t^{\lambda} \log M\left(r_{k}\right) \quad \text { for } \varepsilon \leq t \leq \frac{1}{\varepsilon},
$$

provided $k$ is large enough. 
For a sequence $\left(r_{k}\right)$ satisfying (4.1) we consider, as in [2], the subharmonic functions

$$
u_{k}(z):=\frac{\log \left|f\left(r_{k} z\right)\right|}{\log M\left(r_{k}\right)} \text { and } v_{k}(z):=\frac{\log \left|f\left(r_{k} z\right)-1\right|}{\log M\left(r_{k}\right)} .
$$

Arguing as in [2, p. 97] we can deduce from [11, Thm 4.1.8 and Thm. 4.1.9] or [12, Thm. 3.2.12 and Thm. 3.2.13] that, passing to a subsequence if necessary, the limits

$$
u(z):=\lim _{k \rightarrow \infty} \frac{\log \left|f\left(r_{k} z\right)\right|}{\log M\left(r_{k}\right)} \text { and } v(z):=\lim _{k \rightarrow \infty} \frac{\log \left|f\left(r_{k} z\right)-1\right|}{\log M\left(r_{k}\right)}
$$

exist and are subharmonic in $\mathbb{C}$. Here the convergence is in the Schwartz space $\mathscr{D}^{\prime}$. This implies that we also have convergence in $L_{\text {loc }}^{1}$.

Moreover, the limits in (4.3) have the following properties:

(a) $\max \{u(z), 0\}=\max \{v(z), 0\}$ for all $z \in \mathbb{C}$;

(b) $\{z: u(z)<0\} \cap\{z: v(z)<0\}=\emptyset$;

(c) $u$ is harmonic in $\mathbb{C} \backslash S_{0}$ and $v$ is harmonic in $\mathbb{C} \backslash S_{1}$;

(d) $\max _{|z|=1} u(z)=\max _{|z|=1} v(z)=1$.

If $\left(r_{k}\right)$ is a sequence of Pólya peaks of order $\lambda>0$, then we also have

(e) $u(0)=v(0)=0$;

(f) $\max \{u(z), v(z)\} \leq|z|^{\lambda}$ for all $z \in \mathbb{C}$.

We refer to [2, p. 97] for the deduction of these properties.

Suppose first that $\left(r_{k}\right)$ is a sequence of Pólya peaks of order $\lambda>0$ so that $(a)-(f)$ hold. Note that such a sequence exists by (4.2) since $\rho \geq 1$.

Let $P$ be the set where one of the functions $u$ and $v$ is positive. In view of $(a)$ this set coincides with the set where both functions are positive. Let $z_{0} \in P$. Then $z_{0} \neq 0$ by $(e)$ and since $S_{0} \cap S_{1}=\{0\}$ we deduce from $(c)$ that one of the functions $u$ and $v$ is harmonic in some neighborhood of $z_{0}$. In particular, it is continuous in $z_{0}$ and thus positive in some (possibly smaller) neighborhood of $z_{0}$. Hence $P$ is open.

Let $N$ be the set of points where at least one of the functions $u$ and $v$ is negative. Since subharmonic functions are upper semicontinuous, $N$ is also open.

Thus the complement $E:=\mathbb{C} \backslash(P \cup N)=\{z: u(z)=v(z)=0\}$ is closed. We will show that $E$ has no interior. Suppose to the contrary that $E$ has an interior point $z_{0}$. Without loss of generality we may assume that $z_{0} \notin S_{1}$. By $(c)$, the function $v$ is harmonic in $\mathbb{C} \backslash S_{1}$, which contains a neighborhood of $z_{0}$. It follows that $v(z)=0$ for all $z \in \mathbb{C} \backslash S_{1}$. By $(a)$ we have $u(z) \leq 0$ for all $z \in \mathbb{C} \backslash S_{1}$. If $u\left(z_{1}\right)<0$ for some $z_{1} \in \mathbb{C} \backslash S_{1}$, then $u(z)<0$ for all $z \in \mathbb{C} \backslash S_{1}$ by the maximum principle. This is a contradiction since $u\left(z_{0}\right)=0$ and we assumed that $z_{0} \in \mathbb{C} \backslash S_{1}$. Hence $u(z)=0$ for all $z \in \mathbb{C} \backslash S_{1}$. Since $u$ is harmonic in $\mathbb{C} \backslash S_{0}$ by $(c)$, we find that $u(z)=0$ for all $z \in \mathbb{C}$, a contradiction. Thus $E$ has no interior.

Our next goal is to show that either $N \subset S_{0} \cup S_{1}$ or $N \supset \mathbb{C} \backslash S_{1}$, where the latter case can occur only if $\theta_{1}=\pi$. To this end, let $Q$ be a component of $N$ such that $u(z)<0$ for $z \in Q$. We will show that $\partial Q \subset S_{1}$. In order to do so, suppose that $z_{0} \in \partial Q \backslash S_{1}$. Then $v$ is harmonic in some neighborhood $V$ of $z_{0}$. By $(a)$ and $(b)$ we 
have $v(z)=0$ for $z \in Q$. It follows that $v(z)=0$ for $z \in V$. On the other hand, $V$ also contains a point $z_{1}$ such that $u\left(z_{1}\right)>0$. Thus $v\left(z_{1}\right)=u\left(z_{1}\right)>0$ by $(a)$. This is a contradiction. Thus $\partial Q \subset S_{1}$. If $\theta_{1}<\pi$, then $Q$ cannot contain $\mathbb{C} \backslash S_{1}$ by Lemma 3.1. Hence $Q \subset S_{1}$ if $\theta_{1}<\pi$. But if $\theta_{1}=\pi$, then it is also possible that $Q \supset \mathbb{C} \backslash S_{1}$.

Similarly, if $Q$ is a component of $N$ such that $v(z)<0$ for $z \in Q$, then $\partial Q \subset S_{0}$. Since we assumed that $\theta_{0}<\pi$, this implies together with Lemma 3.1 that $Q \subset S_{0}$. Overall we see that $N \subset S_{0} \cup S_{1}$ or $N \supset \mathbb{C} \backslash S_{1}$, as claimed above. In the latter case, $u(z)<0$ for $z \in \mathbb{C} \backslash S_{1}$.

We first consider the case that $N \subset S_{0} \cup S_{1}$. It follows that $u(z) \geq 0$ and $v(z) \geq 0$ for $z \in \mathbb{C} \backslash\left(S_{0} \cup S_{1}\right)$. Since both $u$ and $v$ are harmonic in $\mathbb{C} \backslash\left(S_{0} \cup S_{1}\right)$ we actually have $u(z)>0$ and $v(z)>0$ for $z \in \mathbb{C} \backslash\left(S_{0} \cup S_{1}\right)$ by the minimum principle. Hence $u(z)=v(z)$ for $z \in \mathbb{C} \backslash\left(S_{0} \cup S_{1}\right)$ by $(a)$. Thus the function

$$
w(z):= \begin{cases}u(z) & \text { if } z \in \mathbb{C} \backslash S_{0}, \\ v(z) & \text { if } z \in \mathbb{C} \backslash S_{1},\end{cases}
$$

is well-defined and harmonic in $\mathbb{C} \backslash\{0\}$. By the removable singularity theorem $[1$, Thm. 2.3] it is harmonic in $\mathbb{C}$. Hence $w$ has the form $w=\operatorname{Re} g$ for some entire function $g$. Since $w(0)=0$ we may choose $g$ such that $g(0)=0$.

Since $\left(r_{k}\right)$ is a sequence of Pólya peaks of order $\lambda>0$, we can deduce from $(f)$ that

$$
w(z)=\operatorname{Re} g(z) \leq|z|^{\lambda}
$$

This implies that $g$ is a polynomial. In fact, $\lambda$ is a positive integer and we have

$$
g(z)=c z^{\lambda}
$$

for some $c \in \mathbb{C}$. Moreover, $|c|=1$ by $(d)$.

In the above reasoning we can take any $\lambda \in\left[\rho_{*}, \rho^{*}\right] \cap(0, \infty)$, but the conclusion gives that $\lambda$ is a positive integer. Together with (4.2) we conclude that

$$
\rho_{*}=\rho^{*}=\rho \in \mathbb{N} \text {. }
$$

Thus the only possible value for $\lambda$ is $\lambda=\rho$. It follows from (4.7) that (4.1) is satisfied for any sequence $\left(r_{k}\right)$ tending to $\infty$. We mention that this kind of argument appears first in [6, Sect. 7] and [7, p. 1209], and it was also used in [2, p. 100].

More precisely, (4.7) implies that for any $\delta>0$ there exist $r_{0}, t_{0}>0$ such that

$$
t^{\rho-\delta} \log M(r) \leq \log M(t r) \leq t^{\rho+\delta} \log M(r) \quad \text { for } r \geq r_{0} \text { and } t \geq t_{0} .
$$

Dropping the assumption that $\left(r_{k}\right)$ is a sequence of Pólya peaks we still have properties $(a)-(d)$, but instead of $(f)$ we can deduce from (4.8) only that

$\left(f^{\prime}\right) \max \{u(z), v(z)\} \leq \begin{cases}|z|^{\rho+\delta} & \text { for }|z| \geq t_{0}, \\ |z|^{\rho-\delta} & \text { for }|z| \leq 1 / t_{0} .\end{cases}$ 
This still yields $(e)$.

Still assuming that the set $N$ where one of the functions $u$ and $v$ is negative is contained in $S_{0} \cup S_{1}$, we again find that the function $w$ defined by (4.4) is harmonic and of the form $w=\operatorname{Re} g$ for some entire function $g$. Instead of (4.5), which was obtained from $(f)$, we now deduce from $\left(f^{\prime}\right)$ that

$$
w(z)=\operatorname{Re} g(z) \leq \begin{cases}|z|^{\rho+\delta} & \text { for }|z| \geq t_{0} \\ |z|^{\rho-\delta} & \text { for }|z| \leq 1 / t_{0}\end{cases}
$$

This implies that $g$ is a polynomial of degree at most $\rho+\delta$ which has a zero of multiplicity at least $\rho-\delta$ at the origin. Choosing $\delta<1$ we again find that $g$ has the form (4.6) with $\lambda=\rho$; that is,

$$
g(z)=c z^{\rho} .
$$

To summarize, every sequence tending to $\infty$ has a subsequence $\left(r_{k}\right)$ such that

$$
\lim _{k \rightarrow \infty} \frac{\log \left|f\left(r_{k} z\right)\right|}{\log M\left(r_{k}\right)}=\operatorname{Re}\left(c z^{\rho}\right) \text { for } z \in \mathbb{C} \backslash S_{0}
$$

while

$$
\lim _{k \rightarrow \infty} \frac{\log \left|f\left(r_{k} z\right)-1\right|}{\log M\left(r_{k}\right)}=\operatorname{Re}\left(c z^{\rho}\right) \text { for } z \in \mathbb{C} \backslash S_{1}
$$

Next we note that $\log |f|=\operatorname{Re}(\log f)$ for a branch $\log f$ of the logarithm. Also, the derivative $h^{\prime}$ of a holomorphic function $h$ can be computed from its real part, via $h^{\prime}=\partial \operatorname{Re} h / \partial x-i \partial \operatorname{Re} h / \partial y$, or via

$$
h^{\prime}(z)=\frac{1}{\pi i} \int_{|\zeta-a|=t} \frac{\operatorname{Re} h(\zeta)}{(\zeta-z)^{2}} d \zeta \text { for }|z-a|<r
$$

if $h$ is holomorphic in $\{z:|z-a| \leq r\}$. We can thus deduce from (4.9) that

$$
\lim _{k \rightarrow \infty} \frac{r_{k} f^{\prime}\left(r_{k} z\right)}{f\left(r_{k} z\right) \log M\left(r_{k}\right)}=c \rho z^{\rho-1} \text { for } z \in \mathbb{C} \backslash S_{0} .
$$

In particular, if $T_{1}$ is a closed subsector of $\mathbb{C} \backslash S_{1}$, then $f^{\prime}$ has only finitely many zeros in $T_{1}$. Applying the same argument to (4.10) yields that $f^{\prime}$ has only finitely many zeros in any closed subsector $T_{0}$ of $\mathbb{C} \backslash S_{0}$. As we may choose $T_{0}$ and $T_{1}$ such that $T_{0} \cup T_{1}=\mathbb{C}$ we conclude that $f^{\prime}$ has only finitely many zeros in $\mathbb{C}$. Since $f$ and hence $f^{\prime}$ have finite order this implies that $f^{\prime}$ has the form $f^{\prime}=p e^{q}$ with polynomials $p$ and $q$. Thus $f$ has the form (1.1).

It remains to consider the case that $N \supset \mathbb{C} \backslash S_{1}$, with $\theta_{1}=\pi$ and $u(z)<0$ for $z \in \mathbb{C} \backslash S_{1}$. We may assume without loss of generality that $S_{1}$ is the left half-plane and $S_{0}=\{z:|\arg z| \leq \pi-\varepsilon\}$ for some $\varepsilon>0$. It follows from $(e)$ and $(f)$ that $u$ 
satisfies the hypotheses of Lemma 3.2. This lemma then yields that $\rho=1$. Since $u$ is harmonic in $C \backslash S_{0}$ by $(c)$, Lemma 3.5 yields that $u$ has the form $u(z)=a z+b$. Since $u(0)=0$ we have $b=0$ and using $(d)$ we see that $|a|=1$ and in fact $a=-1$. Hence $u(z)=-\operatorname{Re}(z)$; that is,

$$
u_{k}(z)=\frac{\log \left|f\left(r_{k} z\right)\right|}{\log M\left(r_{k}\right)} \rightarrow-\operatorname{Re} z .
$$

As before we can now deduce that the limits (4.3) exist not only if $\left(r_{k}\right)$ is chosen as a sequence of Pólya peaks, but in fact for every sequence $\left(r_{k}\right)$ tending to $\infty$. Once this is known, it is not difficult to see that the answer to the question of whether $N \subset S_{0} \cup S_{1}$ or $N \supset \mathbb{C} \backslash S_{1}$ does not depend on the choice of the sequence $\left(r_{k}\right)$.

Thus we only have to deal with the case that (4.11) holds for every sequence $\left(r_{k}\right)$ tending to $\infty$. We will show that this implies that $f$ has the form $f(z)=e^{a z+b}$ with constants $a$ and $b$. In particular, $f$ has the form (1.1).

It follows from (4.11) that there is a curve $\gamma$ tending to $\infty$ near the imaginary axis in both directions such that $|f(z)|=1$ for $z \in \gamma$. Suppose that $f$ has at least one zero. Then $f$ is unbounded on the imaginary axis by Lemma 3.5, applied to the subharmonic function $\log |f|$. Thus there exists a real sequence $\left(y_{k}\right)$ such that

$$
T_{k}:=\left|f\left(i y_{k}\right)\right| \rightarrow \infty
$$

as $k \rightarrow \infty$. Without loss of generality we may assume that $y_{k} \rightarrow+\infty$. Assuming that $T_{k}>1$ there exists $x_{k}>0$ such that $z_{k}:=x_{k}+i y_{k}$ lies on the curve $\gamma$. We have $x_{k}=o\left(y_{k}\right)$ as $k \rightarrow \infty$ by (4.11).

As before it follows from (4.11) with $r_{k}=y_{k}$ by differentiation that

$$
\lim _{k \rightarrow \infty} \frac{y_{k} f^{\prime}\left(y_{k} z\right)}{f\left(y_{k} z\right) \log M\left(y_{k}\right)}=-1 \text { for } z \in \mathbb{C} \backslash S_{0} .
$$

Put $L_{k}:=\left(\log M\left(y_{k}\right)\right) / y_{k}$. It follows from (4.13) that

$$
\frac{1}{2} L_{k} \leq\left|\frac{f^{\prime}(z)}{f(z)}\right| \leq 2 L_{k} \quad \text { for }\left|z-i y_{k}\right| \leq 2 x_{k},
$$

provided $k$ is large enough. Hence

$$
\begin{aligned}
\log T_{k} & =\log \left|f\left(i y_{k}\right)\right|-\log \left|f\left(x_{k}+i y_{k}\right)\right| \\
& =\operatorname{Re}\left(-\int_{0}^{x_{k}} \frac{f^{\prime}\left(x+i y_{k}\right)}{f\left(x+i y_{k}\right)} d x\right) \\
& \leq \int_{0}^{x_{k}}\left|\frac{f^{\prime}\left(x+i y_{k}\right)}{f\left(x+i y_{k}\right)}\right| d x \\
& \leq 2 x_{k} L_{k} .
\end{aligned}
$$

Let now $\gamma_{k}$ be the component of the intersection of $\gamma$ with the disk $\left\{z:\left|z-z_{k}\right| \leq x_{k}\right\}$ that contains $z_{k}$. Then $f \circ \gamma_{k}$ is a curve contained in the unit circle. We have $f^{\prime}(z) \neq 0$ 
for $z \in \gamma_{k}$ by (4.14), provided $k$ is large enough. This implies that $\arg f(z)$ is monotone as $z$ runs through $\gamma_{k}$. Moreover, we have

$$
\operatorname{length}\left(\gamma_{k}\right) \geq 2 x_{k}
$$

and thus

$$
\operatorname{length}\left(f \circ \gamma_{k}\right) \geq 2 x_{k} \inf _{z \in \gamma_{k}}\left|f^{\prime}(z)\right|=2 x_{k} \inf _{z \in \gamma_{k}}\left|\frac{f^{\prime}(z)}{f(z)}\right|
$$

for large $k$. Combining this with (4.14), (4.15) and (4.12) we find that

$$
\text { length }\left(f \circ \gamma_{k}\right) \geq x_{k} L_{k} \geq \frac{1}{2} \log T_{k}>2 \pi
$$

for large $k$. We conclude that $f \circ \gamma_{k}$ wraps around the unit circle at least once. Hence $\gamma_{k}$ contains a 1-point of $f$, contradicting the hypothesis that all 1-points are in the left half-plane.

It follows that $f$ has no zeros. Hence $f$ is of the form $f(z)=e^{a z+b}$.

Proof of Theorem 1.1 Let $f$ be a transcendental entire function for which all but finitely many zeros are in $S_{0}$ while all but finitely many 1-points are in $S_{1}$. Theorem 1.3 yields that $f$ has the form (1.1) with polynomials $p$ and $q$. We show first that the degree of $q$ is even. To see this, let $\phi_{k}$ and $a_{k}$ be given by (2.1) and (2.2) and suppose that $d:=\operatorname{deg}(q)$ is odd, say $d=2 m-1$ with $m \in \mathbb{N}$. First we note that $a_{k} \in\{0,1\}$ for all $k \in\{1, \ldots, d\}$ since otherwise both zeros and 1-points would accumulate at the rays $\arg z=\phi_{k} \pm \pi /(2 d)$.

Now fix $k \in\{1, \ldots, d\}$ and suppose that $a_{k}=0$. Then the 1-points of $f$ accumulate at the ray $\arg z=\phi_{k}+\pi /(2 d)$. Since all but finitely many 1-points are contained in a sector of opening less than $\pi$, they cannot accumulate at the ray $\arg z=\phi_{k}+$ $\pi /(2 d)+\pi=\phi_{k+m}-2 \pi /(2 d)$. Here the index in $\phi_{k}$ is taken modulo $d$; that is, $\phi_{j}=\phi_{k}$ if $j \equiv k(\bmod d)$. Hence the 1-points also do not accumulate at the ray $\arg z=\phi_{k+m}+2 \pi /(2 d)$. It follows that the zeros accumulate at the ray $\arg z=$ $\phi_{k+m}+2 \pi /(2 d)$. Repeating this argument we deduce that the 1-points accumulate at the rays $\arg z=\phi_{k+2 m} \pm 2 \pi /(2 d)=\phi_{k+1} \pm 2 \pi /(2 d)$. Induction now shows that the 1-points accumulate at the rays $\arg z=\phi_{j} \pm 2 \pi /(2 d)$ for all $j \in\{1, \ldots, d\}$, contradicting our hypothesis that they are contained in a sector of opening angle less than $\pi$. Thus $d$ is even.

Suppose first that $d=2$. Without loss of generality we may assume that $\theta_{0}<\pi / 2$. Then, for $k \in\{1,2\}$, the zeros of $f$ cannot accumulate at both rays $\arg z=\phi_{k} \pm \pi / 4$. As explained in Sect. 2, this implies that the 1-points of $f$ accumulate at the rays $\arg z=\phi_{1} \pm \pi / 4$ and $\arg z=\phi_{2} \pm \pi / 4$. This contradicts the assumption that all but finitely many 1-points are in $S_{1}$.

Suppose now that $d \geq 4$. It follows from the hypothesis that there exists an open sector $T$ of opening angle greater than $\pi / 4$ such that $f$ has only finitely many zeros and 1-points in $T$. However, since $d \geq 4$, there exists $k \in\{1, \ldots, d\}$ such that one of 
the rays $\arg z=\phi_{k}+\pi /(2 d)$ and $\arg z=\phi_{k}-\pi /(2 d)$ is contained in $T$. Since the zeros or 1-points accumulate at these rays, this is a contradiction.

Proof of Theorem 1.2 Let again $\phi_{k}$ and $a_{k}$ be given by (2.1) and (2.2), with $d:=\operatorname{deg}(q)$. As in the proof of Theorem 1.1 we have $a_{k} \in\{0,1\}$ for all $k \in\{1, \ldots, d\}$. By hypothesis there exists a closed sector $T$ of opening angle greater than $\pi / 3$ which intersects $H$ and $S$ only in 0 . This implies that $T$ does not intersect any of the rays $\arg z=\phi_{k} \pm \pi /(2 d)$. It follows that $\pi / d>\pi / 3$ and thus $d<3$.

Suppose that $d=2$. Since the 1-points are contained in a half-plane we have $a_{k}=0$ for some $k \in\{1,2\}$. This implies that the zeros accumulate at both rays $\arg z=$ $\phi_{k} \pm \pi / 4$. Hence there are infinitely many zeros not contained in $S$, a contradiction.

It follows that $d=1$. This implies that $f$ has the form given.

Funding Open Access funding provided by Projekt DEAL.

Open Access This article is licensed under a Creative Commons Attribution 4.0 International License, which permits use, sharing, adaptation, distribution and reproduction in any medium or format, as long as you give appropriate credit to the original author(s) and the source, provide a link to the Creative Commons licence, and indicate if changes were made. The images or other third party material in this article are included in the article's Creative Commons licence, unless indicated otherwise in a credit line to the material. If material is not included in the article's Creative Commons licence and your intended use is not permitted by statutory regulation or exceeds the permitted use, you will need to obtain permission directly from the copyright holder. To view a copy of this licence, visit http://creativecommons.org/licenses/by/4.0/.

\section{References}

1. Axler, S., Bourdon, P., Ramey, W.: Harmonic Function Theory. Graduate Texts in Mathematics, vol. 137. Springer, New York (1992)

2. Bergweiler, W., Eremenko, A., Hinkkanen, A.: Entire functions with two radially distributed values. Math. Proc. Camb. Philos. Soc. 165(1), 93-108 (2018)

3. Biernacki, M.: Sur la théorie des fonctions entières. Bull. Acad. Polon. Sci. Lett. Cl. Sci. Math. Nat. Sér. A. 20, 529-590 (1929)

4. Drasin, D., Shea, D.F.: Pólya peaks and the oscillation of positive functions. Proc. Am. Math. Soc. 34, 403-411 (1972)

5. Edrei, A.: Meromorphic functions with three radially distributed values. Trans. Am. Math. Soc. 78, 276-293 (1955)

6. Erëmenko, A.Ė.: A new proof of the Drasin theorem on meromorphic functions of finite order with a maximal sum of defects. I. (Russian) Teor. Funkt. Funkt. Anal. Prilozhen. No. 51, 107-116 (1989). (translation in J. Soviet Math. 52 (1990), no. 6, 3522-3529)

7. Erëmenko, A.: Meromorphic functions with small ramification. Indiana Univ. Math. J. 42(4), 11931218 (1993)

8. Goldberg, A.A., Ostrovskii, I.V.: Value Distribution of Meromorphic Functions. Translations of Mathematical Monographs, vol. 236. American Mathematical Society, Providence (2008)

9. Hayman, W.K., Kennedy, P.B.: Subharmonic Functions, Volume I. London Mathematical Society Monographs, vol. 9. Academic Press, London (1976)

10. Hemke, J.-M.: Recurrence of entire transcendental functions with simple post-singular sets. Fund. Math. 187(3), 255-289 (2005)

11. Hörmander, L.: The Analysis of Linear Partial Differential Operators I. Second edition. Grundlehren der Mathematischen Wissenschaften, vol. 256. Springer, Berlin (1990)

12. Hörmander, L.: Notions of Convexity. Progress in Mathematics, vol. 127. Birkhäuser, Boston (1994)

13. Levin, B.Y.: Lectures on Entire Functions. Translations of Mathematical Monographs, vol. 150. American Mathematical Society, Providence (1996) 
14. Ransford, T.: Potential Theory in the Complex Plane. London Mathematical Society Student Texts, vol. 28. Cambridge University Press, Cambridge (1995)

Publisher's Note Springer Nature remains neutral with regard to jurisdictional claims in published maps and institutional affiliations. 